Braz J Med Biol Res, April 2012, Volume 45(4) 349-356

doi: $10.1590 / \mathrm{S} 0100-879 X 2012007500030$

Involvement of midbrain tectum neurokinin-mediated mechanisms in fear and anxiety

J.C. Brenes, A.C. Broiz, G.S. Bassi, R.K.W. Schwarting and M.L. Brandão

The Brazilian Journal of Medical and Biological Research is partially financed by

\section{贵CNPq}

da Ciência e Tecnologia

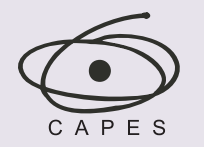
da Educação
Ministério
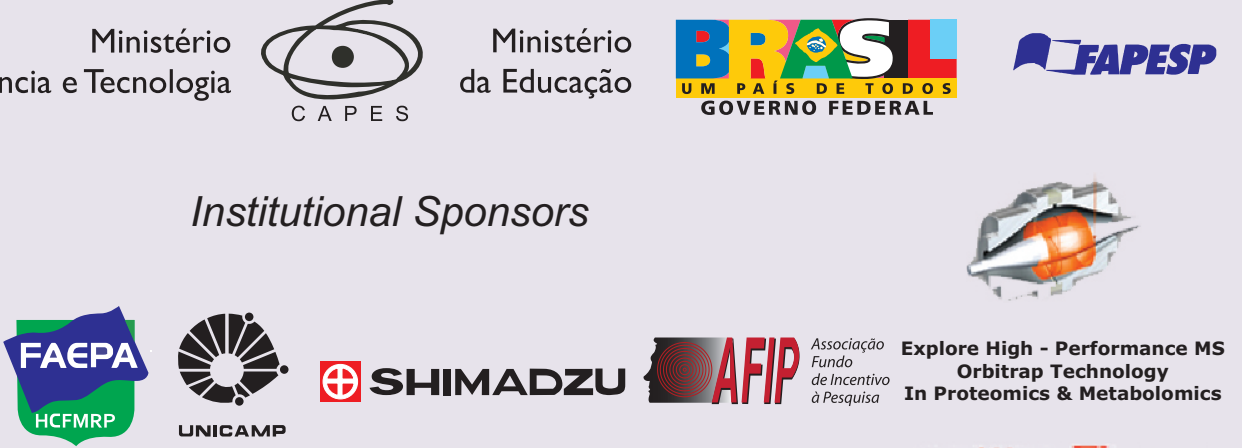

Institutional Sponsors

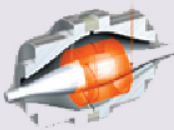

๑ SHIMADZU UNICAMP

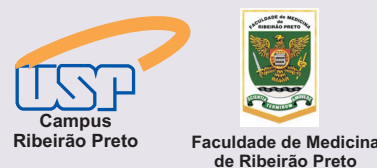
de Ribeirão Preto
de 


\title{
Involvement of midbrain tectum neurokinin-mediated mechanisms in fear and anxiety
}

\author{
J.C. Brenes ${ }^{1}$, A.C. Broiz ${ }^{2,3}$, G.S. Bassi2,3, R.K.W. Schwarting ${ }^{1}$ and M.L. Brandão 2,3 \\ ${ }^{1}$ Experimental and Physiological Psychology, Philipps-University of Marburg, Marburg, Germany \\ ${ }^{2}$ Instituto de Neurociências e Comportamento, Campus USP, Ribeirão Preto, SP, Brasil \\ ${ }^{3}$ Laboratório de Psicobiologia, Faculdade de Filosofia, Ciências e Letras de Ribeirão Preto, \\ Universidade de São Paulo, Ribeirão Preto, SP, Brasil
}

\begin{abstract}
Electrical stimulation of midbrain tectum structures, particularly the dorsal periaqueductal gray (dPAG) and inferior colliculus (IC), produces defensive responses, such as freezing and escape behavior. Freezing also ensues after termination of dPAG stimulation (post-stimulation freezing). These defensive reaction responses are critically mediated by $\mathrm{Y}$-aminobutyric acid and 5-hydroxytryptamine mechanisms in the midbrain tectum. Neurokinins (NKs) also play a role in the mediation of dPAG stimulation-evoked fear, but how NK receptors are involved in the global processing and expression of fear at the level of the midbrain tectum is yet unclear. The present study investigated the role of NK-1 receptors in unconditioned defensive behavior induced by electrical stimulation of the dPAG and IC of male Wistar rats. Spantide (100 pmol/0.2 $\mu \mathrm{L})$, a selective NK-1 antagonist, injected into these midbrain structures had anti-aversive effects on defensive responses and distress ultrasonic vocalizations induced by stimulation of the dPAG but not of the IC. Moreover, intra-dPAG injections of spantide did not influence post-stimulation freezing or alter exploratory behavior in rats subjected to the elevated plus maze. These results suggest that NK-1 receptors are mainly involved in the mediation of defensive behavior organized in the dPAG. Dorsal periaqueductal gray-evoked post-stimulation freezing was not affected by intra-dPAG injections of spantide, suggesting that NK-1-mediated mechanisms are only involved in the output mechanisms of defensive behavior and not involved in the processing of ascending aversive information from the dPAG.
\end{abstract}

Key words: Neurokinin-1 receptors; Dorsal periaqueductal gray; Spantide; Unconditioned fear; Elevated plus maze; Ultrasonic vocalizations

\section{Introduction}

Electrical and chemical stimulation of the dorsal periaqueductal gray (dPAG) and inferior colliculus (IC) causes a characteristic pattern of active defense reactions, which include alertness, freezing, escape responses, and autonomic changes that resemble anxiety disorder $(1,2)$. Much evidence has implicated $y$-aminobutyric acid (GABA), 5-hydroxytryptamine (5-HT), excitatory amino acids, nitric oxide, and opioids in the modulation of defense reactions in these midbrain tectum structures, but less is known about the involvement of neurokinin (NK)-mediated mechanisms in these responses (for a review, see Ref. 3).

Substance $P$ is widely distributed in the brain, including the amygdala, septum, hippocampus, and hypothalamus, and is involved in the regulation of affective behavior and the mediation of stress responses $(4,5)$. Substance $P$ has also been implicated in the mediation of fear in the dPAG $(6,7)$. Local injections of substance $P$ into this region elicit anxiety-like behavior in animals, including conditioned place aversion and a decrease in the time spent in the open arms of the elevated plus maze (EPM), a widely used animal model of anxiety (6-8). Substance $P$ has been shown to be involved in stress-, depression-, and anxiety-related behaviors $(9,10)$. Although the presence of substance $P$ and NK receptors in the IC has been documented in the literature (11), to our knowledge, no study has investigated the possible role of NK-mediated mechanisms in the IC in

Correspondence: M.L. Brandão, Laboratório de Psicobiologia, Faculdade de Filosofia, Ciências e Letras de Ribeirão Preto, Universidade de São Paulo, Ribeirão Preto, SP, Brasil. E-mail: mbrandao@usp.br

Presented at the III Fórum em Neurobiologia do Estresse, Araraquara, SP, Brazil, September 8-10, 2011.

Received November 11, 2011. Accepted February 16, 2012. Available online March 9, 2012. Published April 9, 2012. 
fear and anxiety.

The anxiogenic effects of substance $P$ in the dPAG appear to be mediated by NK-1 receptors $(6,8,12,13)$. NK-1 receptor antagonists may be effective in the treatment of depression in patients with symptoms of anxiety, leading to research efforts aimed at the development of therapies for both depression and anxiety $(9,14)$. The dPAG and IC are involved mainly in the output of defense reactions and processing of aversive information, respectively. Unknown, however, is the extent to which NK-1-mediated mechanisms regulate defense reactions in the $\mathrm{dPAG}$ and IC. Unraveling the role of NK-1-mediated mechanisms in the components of defensive behavior organized in the midbrain tectum will help shed light on the functions of the neural substrates of fear and anxiety. To approach this issue, we will consider several studies that have related each level of defense reactions, such as freezing, escape, and post-stimulation freezing (PSF), with each particular aversive state of the defense reaction. For example, freezing behavior induced by direct stimulation of the dPAG may be considered a preparatory response for escape, and the vigorous, undirected flight elicited by proximal danger may be related to panic. PSF has been related to the processing of aversive information that is relayed to higher brain structures $(3,15$ 17). Here, we examined the effects of local injections of substance $\mathrm{P}$ and the NK-1 receptor antagonist spantide into midbrain structures on freezing, escape, and freezingevoked responses elicited by stimulation of the dPAG and IC in rats. In addition to these behavioral responses, distress ultrasonic vocalizations (USVs) were also recorded during these defensive responses. Several studies from this and other laboratories (18-20) have shown that USVs, especially those emitted in the range of $22-\mathrm{kHz}$, are an important emotional component of the defense reaction. The emission of this type of call is controlled in part by the dPAG, in which substance P-mediated mechanisms seem to play an important role $(7,21)$.

\section{Material and Methods}

\section{Animals}

Male Wistar rats weighing $250-280 \mathrm{~g}$, from the animal house of the Ribeirão Preto Campus, University of São Paulo, were used. They were transported to a local vivarium in the laboratory where they were housed in groups of 4 per cage $(40 \times 30 \times 25 \mathrm{~cm})$. The animals were maintained at constant temperature $\left(22 \pm 1^{\circ} \mathrm{C}\right)$ and on a 12-h/12-h light/ dark cycle (7:00 am-7:00 pm lights on). After surgery, they were group housed (3-5 rats per cage) in Plexiglas-walled cages with free access to food and water throughout the experiment. All experimental procedures were approved by the Committee for Animal Care and Use of the University of São Paulo, Ribeirão Preto Campus (No. 09.1.84.54.7). All efforts were made to minimize animal suffering and to reduce the number of animals used in the study.

\section{Surgery}

The animals were anesthetized with tribromoethanol (250 $\mathrm{mg} / \mathrm{kg}$, ip) and fixed in a stereotaxic frame (David Kopf, USA). The upper incisor bar was set $3.3 \mathrm{~mm}$ below the interaural line such that the skull was horizontal between bregma and lambda. A chemitrode made of a stainless steel guide cannula $(0.6 \mathrm{~mm}$ in outer diameter, $0.4 \mathrm{~mm}$ in inner diameter) was glued to a brain electrode and aimed at the dPAG or IC. The electrode was made of a stainless steel wire $(160 \mu \mathrm{m}$ in diameter) insulated except at the cross-section. The electrode was introduced at a $16^{\circ}$ angle toward the dPAG, with lambda serving as the reference for the following coordinates: anteroposterior $(A P)=0.1$ $\mathrm{mm}$; mediolateral $(\mathrm{ML})=1.9 \mathrm{~mm}$; dorsoventral $(\mathrm{DV})=5.2$ $\mathrm{mm}$. For the IC, the following coordinates were used: AP $=-0.8 \mathrm{~mm} ; \mathrm{ML}=1.2 \mathrm{~mm}$; DV $=5.0 \mathrm{~mm}$. All coordinates were taken from the surface of the skull. For all groups, the electrodes and cannula were fixed to the skull using acrylic resin and two stainless steel screws. The electrode wire was connected to a male pin so that it could be plugged into an amphenol socket at the end of a flexible electrical cable and used for brain stimulation. At the end of surgery, each guide cannula was sealed with a stainless steel wire to protect it from obstruction.

\section{Drugs}

The NK-1 receptor antagonist spantide ([D-Arg ${ }^{1}, \mathrm{D}-$ $\operatorname{Trp}^{7,9}$, Leu ${ }^{11}$ ]-substance $P$ acetate salt) was obtained from Sigma (Brazil), dissolved and diluted to the desired concentration with phosphate-buffered saline (PBS), pH 7.4. Spantide was injected at the dose of $100 \mathrm{pmol} / 0.2 \mu \mathrm{L}$, which was chosen on the basis of previous studies $(7,22)$.

The present study was divided into two experiments with independent groups of animals. The first experiment examined the effects of microinjections of spantide into the dPAG and IC on defense reactions induced by electrical stimulation of these regions. Ultrasonic vocalizations were simultaneously recorded during the expression of the defense responses (freezing, escape and dPAG- or IC-evoked PSF) at baseline and after the drug treatments. In the second experiment, we assessed the effects of intra-dPAG injections of spantide on exploratory behavior in rats subjected to the EPM. The effects of injections of spantide into the IC were not examined in this part of the study because of the absence of effects in the preceding experiment.

\section{Microinjection procedure}

A total volume of $0.2 \mu \mathrm{L}$ was used for the drug injections into the dPAG. Control animals received the same volume of vehicle. The injection needle was a thin dental needle $(0.3 \mathrm{~mm}$ in outer diameter) connected to a $5-\mu \mathrm{L}$ Hamilton syringe by means of a polyethylene tube. It was introduced through the guide cannula until its lower end was $1 \mathrm{~mm}$ below the guide cannula. The solutions were prepared shortly before use and injected into the dPAG $(0.2 \mu \mathrm{L} / \mathrm{min})$, 
driven by an infusion pump (Harvard Apparatus, USA). The displacement of an air bubble inside the polyethylene-10 tubing (Becton-Dickinson, USA) that connected the syringe needle to the intracerebral needle was used to monitor the microinjection. The needle was held in place for an additional $1 \mathrm{~min}$ to maximize diffusion away from the needle tip.

\section{Experiment 1. Effects of spantide on aversive thresholds determined by electrical stimulation of the dPAG and IC}

One week after surgery, the animals were placed in an experimental open field that consisted of a circular enclosure $(60 \times 50 \mathrm{~cm})$ made of transparent Plexiglas. The floor consisted of 15 stainless bars $(2.0 \mathrm{~mm}$ in diameter) spaced $12 \mathrm{~mm}$ apart. The animals were allowed a 5-min period of habituation to the box at the beginning of each session. Thereafter, the brain was stimulated electrically using a sine wave stimulator (Del Vecchio, Brazil). The stimulation current was monitored by measuring the voltage drop across a $1 \mathrm{~K} \Omega$ resistor with an oscilloscope (Philips, Brazil). Brain stimulation (alternating current, $60 \mathrm{~Hz}, 10 \mathrm{~s}$ ) was presented at 1-min intervals with the current intensity increasing by $5-\mu \mathrm{A}$ steps to determine aversive thresholds.

The freezing threshold was operationally defined as the lowest intensity that produced an interruption of ongoing behavior lasting more than $6 \mathrm{~s}$ and attentive postures toward the animals' surroundings (23-25). The current intensity that produced running (galloping) or jumping in two successive trials was considered to be the escape threshold. These measures were confirmed in another consecutive ascending series of electrical stimulation. A cut-off intensity of $150 \mu \mathrm{A}$ (peak-to-peak) was used for electrical stimulation. To investigate the behavioral effects of the electrical stimulation that triggered escape behavior, the animals remained in the experimental box for another 5 min without any stimulation, during which time freezing behavior was recorded. This post-stimulation period is referred to as dPAG PSF $(15,16)$.

After these baseline measurements, each animal was wrapped in a cloth, and a thin dental needle was introduced through the guide cannula until its lower end protruded 1 $\mathrm{mm}$ below the guide cannula. The animals then received either PBS $(\mathrm{N}=8)$ or spantide $(\mathrm{N}=8)$. Ten minutes later, aversive thresholds and the time spent freezing after dPAG or IC stimulation were determined again. The drug dose and waiting time after the injections were selected from previous studies in this laboratory $(7,22)$.

\section{Recording of ultrasound vocalizations}

An Ultra Sound Gate Condenser Microphone (CM 16) that is sensitive to $10-120-\mathrm{kHz}$ frequencies with a flat frequency response between 15 and $30 \mathrm{kHz}( \pm 6 \mathrm{~dB})$ and between 40 and $70 \mathrm{kHz}( \pm 12 \mathrm{~dB})$ was connected to a computer via an Avisoft Ultrasound Gate 416 USB audio device (Avisoft Bioacoustics, Berlin, Germany) such that acoustic data were displayed on the computer in real time (Avisoft Recorder, Avisoft Bioacoustics) and recorded at a sampling rate of $214,285 \mathrm{~Hz}$ in 16-bit format. For acoustical analysis, the recordings were transferred to SASLab Pro (versions 4.3 and 4.52; Avisoft Bioacoustics), and a fast Fourier transformation (FFT) was conducted (512 FFT-length, $100 \%$ frame, Hamming window and $75 \%$ time window overlap). Spectrograms were produced with a 488-Hz frequency resolution and $0.512-\mathrm{ms}$ time resolution. The number of $22-\mathrm{kHz}$ calls was automatically measured using an automatic threshold-based algorithm (55-33-dB threshold) and a hold-time mechanism (10-40-ms hold time). A lower cut-off frequency of $18 \mathrm{kHz}$ was used to reduce background noise outside the relevant frequency band to 0 $\mathrm{dB}$. During each test session, the microphone was placed through a support in the middle of the top surface of the arena approximately $40 \mathrm{~cm}$ above the floor to record the entire spectrum of USVs. A video camera linked to a TV monitor was used to monitor global behavior during the entire recording period.

\section{Experiment 2. Effects of spantide in the EPM}

As a control for the preceding experiments, the effects of treatment with 100 pmol spantide on spontaneous exploratory activity were evaluated. For this purpose, additional groups of rats were tested individually in the EPM. The EPM was made of wood and had two open arms $(50 \times 10 \mathrm{~cm})$ that were perpendicular to two closed arms of the same size with $50-\mathrm{cm}$ high walls. The apparatus was elevated $50 \mathrm{~cm}$ above the floor. To avoid falls, the open arms were surrounded by a 3-mm wooden rim. The apparatus was located inside a room with constant noise $(50 \mathrm{~dB})$. The behavior of the animals was recorded with a video camera (Everfocus, USA) positioned above the maze. The signal was relayed to a monitor in another room via a closed-circuit video camera, which allowed the identification of all forms of behavior. Luminosity at the level of the open arms of the EPM was 20 lux. The maze was cleaned thoroughly after each test with damp and dry cloths. Experimental sessions were conducted between 12:00 and 6:00 pm and lasted 5 $\mathrm{min}$. The rats were placed individually in the center of the EPM facing a closed arm. An observer trained to measure ethological EPM parameters subsequently scored the videotapes using the Observer analysis software developed by Noldus (Amsterdam, The Netherlands). We measured the number of entries into both arms and the time spent on different parts of the maze. An arm entry or exit was defined as all four paws entering into or exiting an arm, respectively. These data were used to calculate the percentage of open arm entries and percentage of time spent in the open arms. A detailed description of the use of the EPM in this laboratory can be found elsewhere (6-8).

\section{Histology}

After the completion of the experiments, the animals 
received an overdose of urethane and were intracardially perfused with saline followed by $4 \%$ buffered formalin. Evans blue (2\%) was then microinjected into the dPAG at the same volume as the drug microinjections to mark the drug injection sites. The brains were removed and maintained in formalin for 1 day and then maintained in $30 \%$ sucrose for another 3 days. Serial $60-\mu \mathrm{m}$ brain sections were cut with a microtome, thaw-mounted on gelatin-coated slides, and Nissl-stained to identify the sites of the injections according to the atlas of Paxinos and Watson (26).

\section{Statistical analysis}

Data are reported as means \pm SEM. In Experiment 1 , aversive threshold differences between pre- and posttreatments and the duration of PSF were analyzed by the Student $t$-test. For the USV analyses, two-way analysis of variance (ANOVA, without repeated measures) was used to assess the effects of spantide and PBS injections into the dPAG and IC. Treatment (PBS or spantide) and condition (baseline and test) were the factors. In Experiment 2, the number of open and closed arm entries and the percentage of time spent in the open and closed arms relative to the total time of the EPM test were analyzed by the Student $t$-test. Differences between multiple means were assessed by the Bonferroni $t$-test. Values of $\mathrm{P}<0.05$ were considered to be statistically significant.

\section{Results}

As the intensity of the current applied to the dPAG and
IC increased, the animals suddenly stopped their ongoing behavior, became immobile, and often urinated and defecated. With higher intensities, this freezing behavior was followed by vigorous running and jumping. The electrode tips and injection sites were situated inside the dorsolateral and dorsomedial divisions of the PAG as well as inside the central nucleus of the IC. These sites were similar to those shown in previous papers published from this laboratory $(6,7,15,16,25,27)$. Although the electrical stimulation applied to the dPAG would activate fibers of passage through this region, the volume of $0.2 \mu \mathrm{L}$ does not spread in a tissue diameter higher than $0.4 \mathrm{~mm}$ in the midbrain tectum (27). This allowed us to assign the effects of drugs specifically to cell bodies of origin in the dPAG.

\section{Experiment 1}

Figure $1 A$ and $B$ show the mean \pm SEM variation in freezing and escape thresholds, respectively, in response to electrical stimulation of the dPAG during the baseline and test phases in the groups of animals injected with PBS and spantide into the dPAG. The Student $t$-test showed that the treatments significantly increased freezing thresholds $\left(t_{1,14}\right.$ $=2.34, \mathrm{P}<0.05)$ and escape thresholds $\left(t_{1,14}=2.17, \mathrm{P}<\right.$ $0.05)$. Figure $1 \mathrm{C}$ shows that no significant effect of treatments on the duration of dPAG-evoked PSF was found $\left(t_{1,14}=0.94, \mathrm{P}>0.05\right)$. The lower row in Figure 1 shows the variations in freezing (Figure 1D) and escape (Figure 1E) thresholds and the duration (Figure 1F) of IC PSF. The freezing thresholds $\left(t_{1,14}=0.34, \mathrm{P}>0.05\right)$, escape thresholds $\left(t_{1,14}=0.67, \mathrm{P}>0.05\right)$, and PSF $\left(t_{1,14}=0.96\right.$,
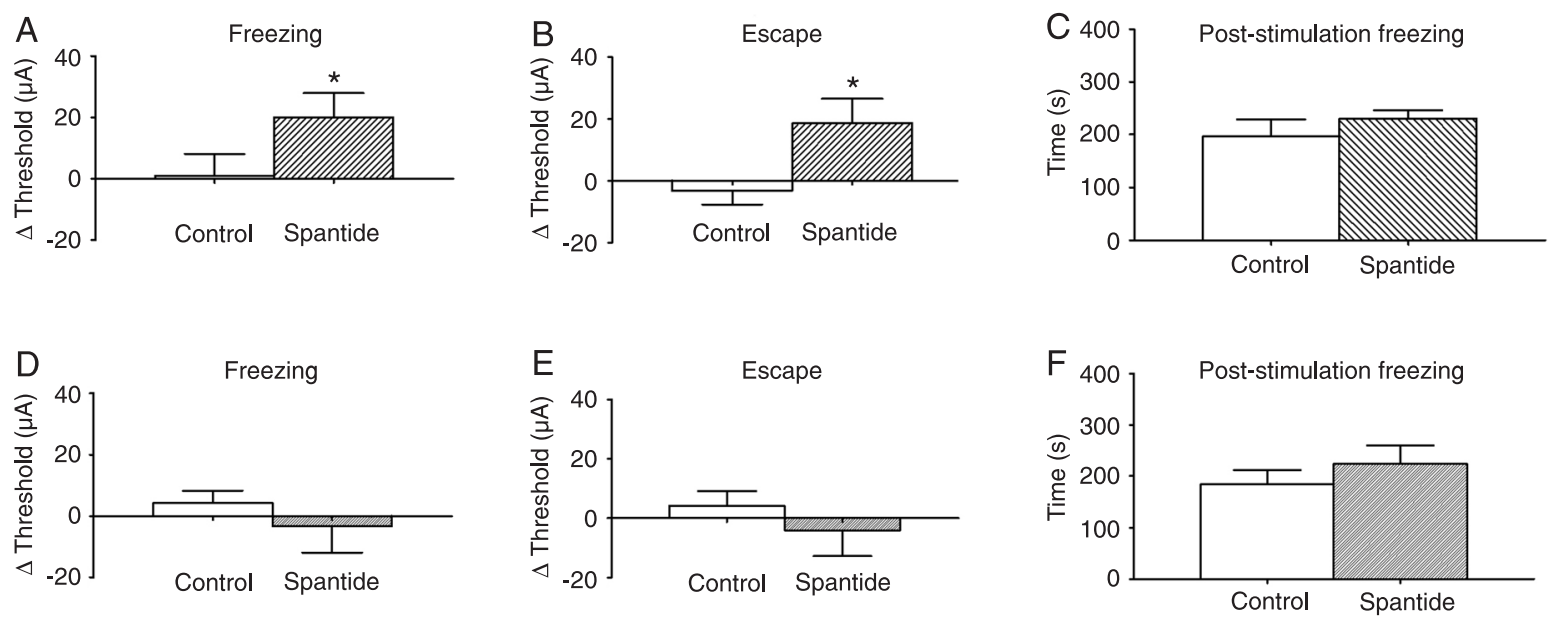

Figure 1. Effect of microinjections of PBS (control) and $100 \mathrm{pmol}$ spantide into the dorsal periaqueductal gray $(A-C)$ and inferior colliculus $(D-F)$ of rats on freezing $(A$ and $D)$ and escape $(B$ and $E$ ) thresholds. The time of post-stimulation freezing is reported in Panels $C$ and $F$. Data are reported as means \pm SEM for $N=8$ rats per group. ${ }^{*} \mathrm{P}<0.05$ compared to control (Student $t$ test). 


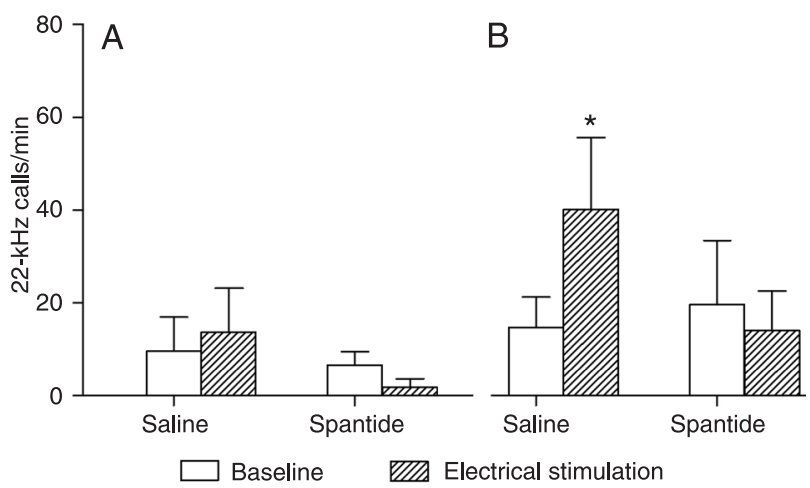

Figure 2. Effects of injection of spantide into the inferior colliculus $(A)$ and the dorsal periaqueductal gray $(B)$ on the number of $22-\mathrm{kHz}$ vocalizations (mean $\pm \mathrm{SEM}$ ) emitted during electrical stimulation of the midbrain tectum. $\mathrm{N}=8$ rats per group. ${ }^{*} \mathrm{P}<$ 0.05 compared to baseline in the control group (two-way ANOVA followed by the Bonferroni test).

$P>0.05)$ did not differ significantly between the groups injected with PBS or spantide into the IC.

USVs were predominantly induced during the electrical stimulation of midbrain tectum structures at the freezing and escape thresholds. Figure 2A shows the number of $22-\mathrm{kHz}$ calls induced by electrical stimulation of the IC in rats that received saline or spantide. Two-way ANOVA revealed no significant effects of treatment or condition on the number of $22-\mathrm{kHz}$ calls and no treatment $x$ condition interaction $\left(F_{1,28}\right.$ $=0.03,0.01,0.015$, respectively, $\mathrm{P}>0.05$ ). Figure $2 \mathrm{~B}$ shows the number of $22-\mathrm{kHz}$ calls induced by electrical stimulation of the $\mathrm{dPAG}$ in rats that received saline or spantide. Two-way ANOVA revealed significant effects of treatment and condition $\left(F_{1,28}=4.63\right.$ and 7.89 , respectively, $\left.P<0.05\right)$ on the number of calls. The treatment $x$ condition interaction was also significant $\left(F_{1,28}=4.63, \mathrm{P}<0.05\right)$. Post hoc comparisons revealed that electrical stimulation increased the number of USVs emitted, and intra-dPAG injections of spantide reduced these USV emissions.

\section{Experiment 2}

Figure 3 shows the effects of control and $100-p m o l$ spantide injections into the dPAG on exploratory activity in the EPM. The Student $t$-test showed that the treatments did not significantly change the number of entries into the open arms $\left(t_{1,14}=1.52, P>0.05\right)$, the number of entries into the closed arms $\left(t_{1,14}=0.08, \mathrm{P}>0.05\right)$, or the time spent in the open arms $\left(t_{1,14}=1.44, \mathrm{P}>0.05\right)$.

\section{Discussion}

The present study extends the analysis of the involvement of NK in defense reactions by comparing the effects of the NK-1 receptor antagonist spantide into the dPAG and IC. Substance $P$ is involved in several behavioral
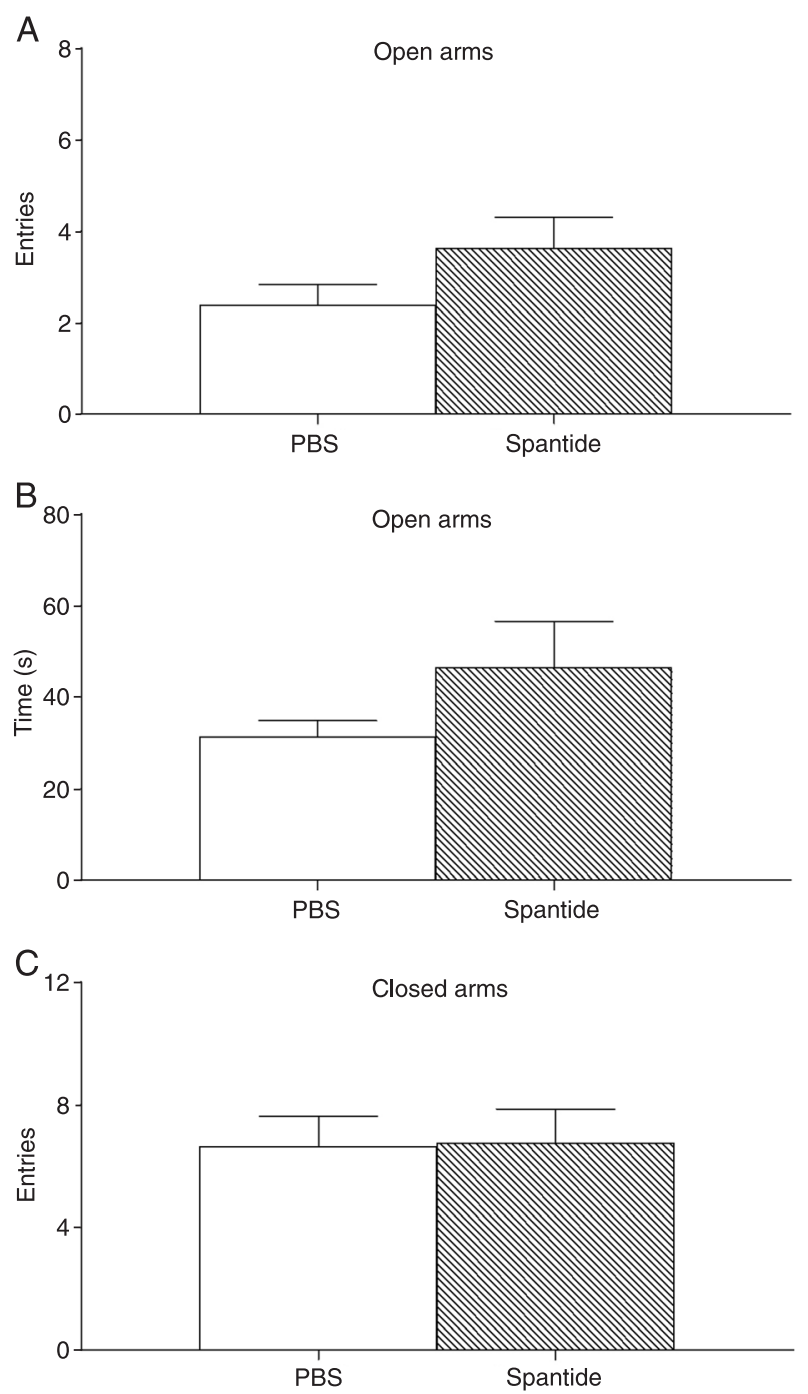

Figure 3. Effects of injections of phosphate-buffered saline (PBS) and $100 \mathrm{pmol}$ spantide into the dorsal periaqueductal gray on the exploratory behavior of rats subjected to the elevated plus maze. $A$ and $C$, Number of entries into the open and closed arms of the maze. $B$, Time spent in the open arms of the maze. Data are reported as means \pm SEM for $N=8$ rats per group (Student $t$-test).

processes, such as reinforcement, learning, memory, and pain (28-31). Substance P-mediated mechanisms involved in stress, depression-, and anxiety-related behaviors have recently received considerable attention $(9,10)$. Regarding the neural substrates of fear and anxiety, we sought to investigate the extent to which NK mechanisms regulate the expression of fear behavior in the dPAG and IC. Substantial concentrations of substance $P$ have been found in the dPAG (32), and substance $P$ has been shown to be a mediator of defensive behavior. Unknown, however, is the extent to which these mechanisms operate in the IC, another structure of the brain aversion system. 
Substance $P$ binds to the three known NK receptors but has greater affinity for the NK-1 receptor (33). The observation that NK-1 receptor antagonists may be effective in the treatment of depression in patients with symptoms of anxiety has led to research efforts aimed at the development of NK-related therapeutics for both depression and anxiety $(9,14,34,35)$. Several studies have shown that the anxiogenic-like effects of substance $P$ in the dPAG appear to be mediated by NK-1 receptors $(7,8,12,13)$. Infusion of the NK-1 agonist SAR-MET-SP into the dPAG had anxiogeniclike and antinociceptive effects in the EPM (7). These data suggest that NK-1 mechanisms in the dPAG are involved in the processing of proximal threatening stimulations or intense fear. NK-1 receptors appear to be involved in the aversive effects of substance $P$ in the dPAG, but the role of NK-2 and NK-3 receptors in this region in the modulation of fear is still unclear. Previous studies from this laboratory showed that NK-1-mediated mechanisms, but not NK-3, are involved in the mediation of defensive behavior elicited by stimulation of the dPAG (22). The anti-aversive effects of spantide in the present study support the hypothesis that $\mathrm{NK}-1$ receptors in the $\mathrm{dPAG}$ play a pro-aversive role and corroborate and extend the proposed involvement of the NK system in the modulation of defensive behavior in this region.

NK-1 receptors in the dPAG do not appear to be involved in the modulation of dPAG PSF because no effect was observed after spantide injections into the dPAG. Dorsal periaqueductal gray PSF is not a conditioned response to the context where electrical stimulation of the dPAG was administered. Previous studies from this laboratory using the context shift procedure have shown that freezing behavior persists when the animal is placed in a different context soon after dPAG stimulation $(15,16)$. Post-stimulation freezing is regulated by mechanisms that are different from dPAG-evoked fear. Electrolytic lesions or muscimol-induced inactivation of the amygdaloid complex reduced dPAG PSF but did not affect dPAG-evoked freezing and escape $(17,36)$. The dPAG PSF depends on an ascending path from the dPAG to rostral brain structures probably relayed by the laterodorsal nucleus of the thalamus since dPAG-evoked post-stimulation freezing behavior causes simultaneous Fos-labeling in this thalamic nucleus (for a review, see Ref. 3). Thus, ascending dPAG efferents appear to be activated during this condition and the lack of change in freezing and escape thresholds observed in amygdala-lesioned or -inactivated animals may be linked to the fact that the dPAG itself contains neural substrates of the production of unconditioned fear. Thus, although dPAG-evoked freezing and dPAG PSF are interrelated, they appear to have different neural substrates, similar to the differential substrates of dPAG-evoked freezing and escape $(17,25,37)$. Probably, dPAG integrates sensory information by transmitting this information to the amygdala, which allows the recognition of threatening stimuli in fear-experienced animals.
The lack of effect of intra-IC injections of spantide on defense reactions induced by stimulation of this midbrain area needs to be discussed in the context of sensorimotor gating of the defense reaction. The dPAG is generally recognized as an output center for defensive behavior and processes aversive information. Thus, whereas freezing and escape responses are the result of aversive stimulation of this area and representative of the expression of the defense reaction, dPAG PSF has been related to the processing of aversive information in higher brain structures, including the amygdala. In contrast, the IC appears to be a part of the entire defense process in a way that is different from the dPAG. The IC is more involved with the sensory output component of the defense reaction. NK-mediated mechanisms do not appear to mediate this sensory component of the defense reaction. Dorsal periaqueductal gray PSF and fear responses induced by electrical stimulation of the IC were not affected by local injections of spantide. These data suggest that NK-mediated mechanisms are not involved in the processing of ascending aversive information from the midbrain tectum. Only the motor component of the defense reaction triggered by dPAG stimulation was sensitive to spantide injection.

NK-1 and NK-3 receptors are widely distributed in the brain, particularly in most limbic structures, and NK-2 receptors are found only in a few structures that are not directly related to the integration of emotional behavior (38). NK-3 receptors have been associated with locomotor activity, pain processing, and promnestic effects (22). In contrast, substance $P$ and NK-1 agonists exert anxiogenic effects when applied locally to the dPAG $(7,8,39)$. However, we found no effect of the selective NK-1 antagonist spantide on exploratory behavior in rats subjected to the EPM, suggesting discrepant results. Intra-dPAG injections of spantide did not affect the conflict expressed by rats when they are confronted with the need to explore a novel environment and to avoid unknown areas, which are characteristic behaviors observed in this test. One possibility of reconciling this discrepancy is to consider that two dissociable processes are recruited in the EPM: i) anxiogenic stimuli associated with the EPM activate pathways in this region that ascend to higher structures of the brain aversion system, and ii) the height and open spaces of the EPM trigger output motor mechanisms of the defense reaction that exist in the dPAG. The former process is not mediated by NK-1 mechanisms. The neural substrates that underlie these processes are responsible for the acquisition of aversive information. Since substance P-mediated mechanisms are mainly involved in the output processes related to the defense reaction, this may explain why spantide did not affect PSF-evoked freezing and did not exert any effects on exploratory behavior in the EPM in rats that received injections into the dPAG. An experiment using the EPM test is under way in this laboratory to examine the effects of injections of spantide into the IC, a structure of the brain 
aversion system that is mainly involved in the transfer of aversive information to other structures and is not an output center for the defense reaction, in contrast to the dPAG (for a review, see Ref. 40). With regard to the latter process, NK-1 receptors modulate the defense mechanisms of the dPAG only when they are called into play by the presence of aversive stimuli, i.e., this mediation is phasic. Substance $P$ may mimic the effects of anxiogenic stimuli and activates these neural mechanisms, but NK-1 antagonists do not produce any effect by their own. 5-HT mechanisms are also considered to modulate phasically the neural substrates of defense in the midbrain tectum since 5-HT receptor agonists counteract, but 5-HT antagonists have no effects, the aversion induced by stimulation of these structures (for a review, see Ref. 2). This is in sharp contrast to GABA mechanisms, which tonically modulate the descending pathways associated with the defensive behavior. When these neural mechanisms are under tonic control, GABA antagonists do release the defense reaction (for a review, see Ref. 40). Defensive responses to aversive stimulation, such as USVs, that are clearly mediated by these output mechanisms in the dPAG (20) are sensitive to the effects of NK-1 receptor antagonists (7). NK-1-mediated mechanisms operate only during the expression of the defense reaction.

\section{References}

1. Brandão ML, Tomaz C, Borges PC, Coimbra NC, Bagri A. Defense reaction induced by microinjections of bicuculline into the inferior colliculus. Physiol Behav 1988; 44: 361365.

2. Brandão ML, Anseloni VZ, Pandossio JE, De Araujo JE, Castilho VM. Neurochemical mechanisms of the defensive behavior in the dorsal midbrain. Neurosci Biobehav Rev 1999; 23: 863-875.

3. Brandão ML, Zanoveli JM, Ruiz-Martinez RC, Oliveira LC, Landeira-Fernandez J. Different patterns of freezing behavior organized in the periaqueductal gray of rats: association with different types of anxiety. Behav Brain Res 2008; 188: 1-13.

4. Rigby M, O'Donnell R, Rupniak NM. Species differences in tachykinin receptor distribution: further evidence that the substance $\mathrm{P}$ (NK1) receptor predominates in human brain. J Comp Neurol 2005; 490: 335-353.

5. Nagano M, Saitow F, Haneda E, Konishi S, Hayashi M, Suzuki $\mathrm{H}$. Distribution and pharmacological characterization of primate NK-1 and NK-3 tachykinin receptors in the central nervous system of the rhesus monkey. Br J Pharmacol 2006; 147: 316-323.

6. De Araujo JE, Huston JP, Brandão ML. Opposite effects of substance $\mathrm{P}$ fragments $\mathrm{C}$ (anxiogenic) and $\mathrm{N}$ (anxiolytic) injected into dorsal periaqueductal gray. Eur $\mathrm{J}$ Pharmacol 2001; 432: 43-51.

7. Bassi GS, Nobre MJ, De Araujo JE, Brandão ML. Anxiogenic effects of activation of NK-1 receptors of the dorsal periaqueductal gray as assessed by the elevated plus-maze, ultrasound vocalizations and tail-flick tests. Neuropeptides
However, substantial research remains to be done to clarify the precise mechanism of action of these compounds and dissociate the processing of the defense reaction from the motor output of the defense reaction.

The present study extends the analysis of the involvement of NK in defense reactions by comparing the effects of the NK-1 receptor antagonist spantide into the dPAG and IC. Dorsal periaqueductal gray PSF was unaffected by NK-1 and NK-3 drugs injected into the dPAG (22), suggesting that ascending aversive information from the dPAG to higher structures in the brain is not controlled by NK-mediated mechanisms. Taken together, the results from this laboratory support the hypothesis that NK-1 receptors mediate defensive behaviors induced by electrical stimulation of the dPAG in rats.

\section{Acknowledgments}

Research supported by FAPESP (\#2011/00041-3). A.C. Broiz and G.S. Bassi are recipients of scholarships from CAPES and CNPq, respectively. M.L. Brandão is a research fellow of CNPq. J.C. Brenes was funded by the Deutscher Akademischer Austausch Dienst (DAAD) and the University of Costa Rica.
2007; 41: 365-374.

8. De Araujo JE, Huston JP, Brandão ML. Place aversion induced by microinjections of $\mathrm{C}$-fragment of substance $\mathrm{P}$ into the dorsal periaqueductal gray of rats is mediated by tachykinin NK1 receptors. Peptides 2001; 22: 1447-1452.

9. Kramer MS, Cutler N, Feighner J, Shrivastava R, Carman $\mathrm{J}$, Sramek JJ, et al. Distinct mechanism for antidepressant activity by blockade of central substance $\mathrm{P}$ receptors. Science 1998; 281: 1640-1645.

10. Bondy B, Baghai TC, Minov C, Schule C, Schwarz MJ, Zwanzger $\mathrm{P}$, et al. Substance $\mathrm{P}$ serum levels are increased in major depression: preliminary results. Biol Psychiatry 2003; 53: 538-542.

11. Hafidi A, Beurg M, Bouleau Y, Dulon D. Comparative distribution of NK1, NK2, and NK3 receptors in the rat brainstem auditory nuclei. Brain Res 2002; 947: 299-306.

12. Mongeau R, De Oca BM, Fanselow MS, Marsden CA. Differential effects of neurokinin-1 receptor activation in subregions of the periaqueductal gray matter on conditional and unconditional fear behaviors in rats. Behav Neurosci 1998; 112: 1125-1135.

13. Duarte FS, Testolin R, De Lima TC. Further evidence on the anxiogenic-like effect of substance $P$ evaluated in the elevated plus-maze in rats. Behav Brain Res 2004; 154: 501-510.

14. Santarelli L, Gobbi G, Debs PC, Sibille ET, Blier P, Hen R, et al. Genetic and pharmacological disruption of neurokinin 1 receptor function decreases anxiety-related behaviors and increases serotonergic function. Proc Natl Acad Sci U S A 2001; 98: 1912-1917. 
15. Vianna DM, Graeff FG, Landeira-Fernandez J, Brandão $\mathrm{ML}$. Lesion of the ventral periaqueductal gray reduces conditioned fear but does not change freezing induced by stimulation of the dorsal periaqueductal gray. Learn Mem 2001; 8: 164-169

16. Vianna DM, Landeira-Fernandez J, Brandão ML. Dorsolateral and ventral regions of the periaqueductal gray matter are involved in distinct types of fear. Neurosci Biobehav Rev 2001; 25: 711-719.

17. Martinez RC, de Oliveira AR, Brandão ML. Conditioned and unconditioned fear organized in the periaqueductal gray are differentially sensitive to injections of muscimol into amygdaloid nuclei. Neurobiol Learn Mem 2006; 85: 58-65.

18. Wohr M, Borta A, Schwarting RK. Overt behavior and ultrasonic vocalization in a fear conditioning paradigm: a doseresponse study in the rat. Neurobiol Learn Mem 2005; 84 : 228-240.

19. Tomazini FM, Reimer A, Tomazini FM, Brandão ML. Opposite effects of short- and long-duration isolation on ultrasonic vocalization, startle and prepulse inhibition in rats. $J$ Neurosci Methods 2006; 153: 114-120.

20. Litvin Y, Blanchard DC, Blanchard RJ. Rat $22 \mathrm{kHz}$ ultrasonic vocalizations as alarm cries. Behav Brain Res 2007; 182: 166-172.

21. Leman S, Dielenberg RA, Carrive P. Effect of dorsal periaqueductal gray lesion on cardiovascular and behavioural responses to contextual conditioned fear in rats. Behav Brain Res 2003; 143: 169-176.

22. Bassi GS, Broiz AC, Gomes MZ, Brandão ML. Evidence for mediation of nociception by injection of the NK-3 receptor agonist, senktide, into the dorsal periaqueductal gray of rats. Psychopharmacology 2009; 204: 13-24.

23. Coimbra NC, Brandão ML. GABAergic nigro-collicular pathways modulate the defensive behaviour elicited by midbrain tectum stimulation. Behav Brain Res 1993; 59: 131-139.

24. Maisonnette SS, Kawasaki MC, Coimbra NC, Brandão ML. Effects of lesions of amygdaloid nuclei and substantia nigra on aversive responses induced by electrical stimulation of the inferior colliculus. Brain Res Bull 1996; 40: 93-98.

25. Oliveira LC, Broiz AC, de Macedo CE, Landeira-Fernandez J, Brandão ML. 5-HT2 receptor mechanisms of the dorsal periaqueductal gray in the conditioned and unconditioned fear in rats. Psychopharmacology 2007; 191: 253-262.

26. Paxinos G, Watson C. The rat brain in stereotaxic coordinates. San Diego: Academic Press; 1997.

27. Ferreira-Netto $\mathrm{C}$, Borelli KG, Brandão ML. Distinct Fos expression in the brain following freezing behavior elicited by stimulation with NMDA of the ventral or dorsal inferior colliculus. Exp Neurol 2007; 204: 693-704.

28. Huston JP, Hasenohrl RU. The role of neuropeptides in learning: focus on the neurokinin substance P. Behav Brain Res 1995; 66: 117-127.

29. Hasenohrl RU, Souza-Silva MA, Nikolaus S, Tomaz C, Brandão ML, Schwarting RK, et al. Substance $P$ and its role in neural mechanisms governing learning, anxiety and functional recovery. Neuropeptides 2000; 34: 272-280.

30. Drew GM, Mitchell VA, Vaughan CW. Postsynaptic actions of substance $P$ on rat periaqueductal grey neurons in vitro. Neuropharmacology 2005; 49: 587-595.

31. Chahl LA. Tachykinins and neuropsychiatric disorders. Curr Drug Targets 2006; 7: 993-1003.

32. Li YQ, Jia HG, Rao ZR, Shi JW. Serotonin-, substance P- or leucine-enkephalin-containing neurons in the midbrain periaqueductal gray and nucleus raphe dorsalis send projection fibers to the central amygdaloid nucleus in the rat. Neurosci Lett 1990; 120: 124-127.

33. Mantyh PW. Neurobiology of substance $P$ and the NK1 receptor. J Clin Psychiatry 2002; 63 (Suppl 11): 6-10.

34. Ranga K, Krishnan R. Clinical experience with substance $P$ receptor (NK1) antagonists in depression. J Clin Psychiatry 2002; 63 (Suppl 11): 25-29.

35. Quartara L, Altamura M. Tachykinin receptors antagonists: from research to clinic. Curr Drug Targets 2006; 7: 975992.

36. Oliveira LC, Nobre MJ, Brandão ML, Landeira-Fernandez $\mathrm{J}$. Role of amygdala in conditioned and unconditioned fear generated in the periaqueductal gray. Neuroreport 2004; 15 : 2281-2285

37. Broiz AC, Oliveira LC, Brandão ML. Regulation of conditioned and unconditioned fear in rats by $5-\mathrm{HT} 1 \mathrm{~A}$ receptors in the dorsal periaqueductal gray. Pharmacol Biochem Behav 2008; 89: 76-84.

38. Ebner K, Sartori SB, Singewald N. Tachykinin receptors as therapeutic targets in stress-related disorders. Curr Pharm Des 2009; 15: 1647-1674.

39. Aguiar MS, Brandão ML. Effects of microinjections of the neuropeptide substance $P$ in the dorsal periaqueductal gray on the behaviour of rats in the plus-maze test. Physiol Behav 1996; 60: 1183-1186.

40. Brandão ML, Borelli KG, Nobre MJ, Santos JM, AlbrechetSouza L, Oliveira AR, et al. Gabaergic regulation of the neural organization of fear in the midbrain tectum. Neurosci Biobehav Rev 2005; 29: 1299-1311. 